\title{
Incorporating next-to-leading order matrix elements for hadronic diboson production in showering event generators
}

\author{
Matt Dobbs* \\ Department of Physics and Astronomy, University of Victoria, P.O. Box 3055, Victoria, British Columbia, Canada V8W 3P6
}

(December 7, 2018)

\begin{abstract}
A method for incorporating information from next-to-leading order QCD matrix elements for hadronic diboson production into showering event generators is presented. In the hard central region (high jet transverse momentum) where perturbative QCD is reliable, events are sampled according to the first order tree level matrix element. In the soft and collinear regions next-toleading order corrections are approximated by calculating the differential cross section across the phase space accessible to the parton shower using the first order (virtual graphs included) matrix element. The parton shower then provides an all-orders exclusive description of parton emissions. Events generated in this way provide a physical result across the entire jet transverse momentum spectrum, have next-to-leading order normalization everywhere, and have positive definite event weights. The method is generalizable without modification to any color singlet production process.
\end{abstract}

24.10.Lx, 14.70.-e, 12.38.-t

Keywords: Monte Carlo simulations, Gauge bosons, Quantum chromodynamics, Parton shower

\section{INTRODUCTION}

As high energy collider physics moves towards the TeV frontier, the simulation of collision events at higher orders becomes increasingly important. For the case of hadronic diboson production, higher order corrections are largest in the regions where the physics is most interesting. Event rate enhancements at next-to-leading order in QCD (NLO) have a spoiling effect on the confidence limits for anomalous triple gauge boson couplings, wash out the radiation zero in $W \gamma$ production [1] and approximate radiation zero in $W Z$ production [2], and contribute to the background rate for new physics channels including heavy Higgs boson searches and Supersymmetry signatures.

Until recently, general purpose event generators have included leading order (LO) matrix elements only. An exclusive (meaning all of the parton emissions are explicitly enumerated in the event record) all orders description of multiple emissions is provided by the parton shower approach [3, th which is valid in the regions of soft or collinear emissions, but is not accurate for well-separated partons. The shower occurs with unit probability and so does not alter the cross section which remains leading order. Two of the general purpose showering Monte Carlo programs, HERWIG [5] and PYTHIA [6], have implemented matrix element corrections [7 9] to the parton shower algorithms using the first order tree level matrix element (the one-loop virtual graphs are not included). The corrections are successful in improving the agreement with data in distribution shapes, especially at high transverse momentum. The normalization, however, is everywhere leading order and the overall event rate is usually obtained by normalizing to the data or applying a constant factor obtained from NLO calculations.

In contrast, next-to-leading (fixed) order Monte Carlo integration programs such as the NLO calculations available for $W Z$ production 19 13] provide a first order prediction of the cross section and a good description of the hardest emission. However, these programs frequently generate events with negative weights in certain phase space regions, do not address multiple emission, and give a poor (indeed sometimes unphysical) description in the region of very small jet transverse momentum where perturbative QCD is unreliable. In this small transverse momentum region, the effects of multiple emissions can be resummed (first proposed by [14]) giving a better prediction of inclusive quantities. However, the standard resummation formalism does not describe the exclusive set of partons in the event.

\footnotetext{
*Electronic Mail Address: matt.dobbs@cern.ch

${ }^{1}$ An example of an inclusive quantity is the color singlet system transverse recoil against all parton emissions $\left(P_{\mathrm{WZ}}^{T}\right.$ for the case of $W Z$ production). Inclusive means the contribution from individual emissions is not available, only the contribution summed over all emissions is known. A LO cross section is an inclusive prediction of the event rate. After applying a parton shower algorithm to a LO event, the description becomes exclusive because the enumeration of each parton emission is contained in the event record.
} 
In Ref. 15] a resummed treatment of hadronic $Z$-pair production $p p \rightarrow Z Z$, is given and a comparison is made with the PYTHIA showering event generator. In the region of low $Z$-pair transverse momentum $P_{\mathrm{ZZ}}^{T}$, the resummed spectrum agrees reasonably well with the results from the parton shower approach.

The combination of NLO matrix elements with parton showering algorithms is a natural way to proceed and would provide an exclusive Monte Carlo description of collision events. This goal has two primary challenges: (1) A probabilistic interpretation of NLO matrix elements is difficult to accomplish on account of the delicate cancellations which occur between the virtual and real emission graphs. This generally results in event weights which may be both positive or negative. (2) The parton shower algorithm can generate emissions which are harder than the first (fixed order) emission resulting in a double counting of hard emissions. Both of these drawbacks are accentuated in the region of small inclusive jet transverse momentum.

Ref. 16] addresses the first challenge for the special case of hadronic $W Z$ production with leptonic decays. For each event, a volume of low transverse momentum $\left(P^{T}\right)$ parton emissions (defined by a $P^{T}$ cutoff and assumed to be unobservable by a detector) is integrated numerically keeping the observable quantities (the charged lepton momenta and missing transverse momentum) fixed. The integration volume is generated by allowing the missing transverse momentum to arise from any combination of invisible neutrino and low $P^{T}$ emission. Such an integration ensures one doesn't "look into" the region of small inclusive jet transverse recoil $P_{\mathrm{WZ}}^{T}$, where the fixed order calculation is unreliable. The resultant events can be interpreted probabilistically (event weights are positive definite) and the NLO predictions for observable distributions are preserved. The drawback is that by integrating the volume of jets the specific jet configuration is lost-determining which configuration is most probable brings back the problem of negative weights. This, and the discontinuity caused by the $P^{T}$ cutoff, makes the subsequent application of a parton shower algorithm difficult.

In the present study the positive features of the Ref. [16] algorithm are combined with the matrix element corrections approach. The phase space is divided into two complementary regions: the region accessible to the parton shower ("parton shower region") and the hard central region which is not accessible to the parton shower ("Dead Zone region"). The treatment of the Dead Zone region in this study follows Ref. [8], wherein matrix element corrections to single vector boson production are applied within the framework of the HERWIG program. The extension to diboson production is trivial, the vector boson mass and momentum in Ref. [8] is everywhere replaced by the diboson system mass and momentum. The parton shower region serves as a phase space volume over which emissions can be integrated in a treatment that closely follows Ref. 16. This provides a means of including information from the full NLO matrix element (including one-loop graphs) such that the normalization is NLO and the distributions reproduce the NLO shapes in the regions where the NLO prediction is expected to be reliable. In the low inclusive jet transverse momentum region, where multiple emissions are important and the fixed order prediction becomes unreliable, the distribution shapes are dominated by the all orders parton shower provided by HERWIG (though the normalization remains NLO). In this way events generated with the algorithm described here provide an exclusive description of the jet structure while giving a physical result across the full jet transverse momentum spectrum and providing NLO normalization.

The method described in this paper applies to any hadronic color singlet production process. The specific process of $p p \rightarrow W^{+} Z$ production with vector boson decays to electron/muon-type leptons at the Large Hadron Collider (LHC) energy of $14 \mathrm{TeV}$ is chosen to illustrate the method. The input parameters for this study are $\alpha_{E M}\left(M_{Z}\right)=\frac{1}{128}$, $\sin ^{2} \theta_{W}=0.23, \alpha_{s}\left(M_{Z}\right)=0.1116, M_{W}=80.396 \mathrm{GeV}, M_{Z}=91.187 \mathrm{GeV}$, Cabibbo angle $\cos \theta_{\text {Cabibbo }}=0.975$ with no 3rd generation mixing, and the factorization scale $Q$ is taken to be the hard process mass $\sqrt{\hat{s}}$. Vector boson branching ratios to leptons are taken as $\operatorname{Br}\left(Z \rightarrow l^{+} l^{-}\right)=3.36 \%, \operatorname{Br}\left(W^{ \pm} \rightarrow l^{ \pm} \nu\right)=10.8 \%$. The CTEQ4M [17] structure functions are used with the $b$ quark contribution taken as zero. Matrix elements (LO and NLO) are evaluated with the Baur-Han-Ohnemus (BHO) package [10] and HERWIG is employed for the parton cascade, hadronization, and decays.

In the section which follows, the implementation of matrix element corrections for diboson production in the HERWIG style are presented and a comparison is made between LO with parton shower, NLO, and matrix element corrected distributions. The method for incorporating the full NLO matrix element in the HERWIG showering event generator is presented in Sec. III and is compared with matrix element corrected and NLO distributions. Conclusions are given in the final section.

\section{MATRIX ELEMENT CORRECTIONS}

The parton level Born process for hadronic $W Z$ production is $q \bar{q}^{\prime} \rightarrow W Z$. The kinematics describing this process are given the label 'n-body' and the label '(n+1)-body' is reserved for the first order tree processes $q \bar{q}^{\prime} \rightarrow W Z g$, $q g \rightarrow W Z q^{\prime}$, and $g \bar{q} \rightarrow W Z \bar{q}^{\prime}$.

Given the kinematics of an n-body event as input, a showering Monte Carlo program uses backwards evolution to trace the hard scattering partons back from the hard vertex into the original incoming hadrons while keeping track 
of the distribution of emitted partons (refer to Ref. 18 for a good review). For the case of the HERWIG program, the phase space accessible to the parton shower (the parton shower region) is a subset of the full ( $\mathrm{n}+1)$-body phase space. The boundary of the Dead Zone region inaccessible to the HERWIG parton shower is defined by [8]

$$
\frac{7-\sqrt{17}}{2} M^{2}<\hat{s}<s
$$

and

$$
\left|\cos \hat{\theta}_{j}\right|<1-\frac{3-\sqrt{1+8 \frac{M^{2}}{\hat{s}}}}{\frac{\hat{s}}{M^{2}}-1}
$$

where $M$ is the mass of the color singlet object ( $W Z$ system in this case), $\sqrt{\hat{s}}$ is the mass of the color singlet and parton emission system $(W Z j), \sqrt{s}$ is the machine energy, and $\hat{\theta}_{j}$ is the angle of the emission with respect to the beam in the center of mass frame. The partition of phase space in the $\hat{s} / M^{2}$ vs. $\cos \hat{\theta}_{j}$ plane is shown in Figure 2. The parton shower and Dead Zone regions are precisely complementary, so there can be no double counting when sampling the regions separately. The Dead Zone is the region of hard central emissions and can be populated by sampling the first order tree level matrix element within the boundaries defined in Eqs. 1 and 2. Adding the Dead Zone phase space in this manner to the showering event generator simulation is termed 'hard matrix element corrections'. For single boson production the improvement in the boson recoil transverse momentum spectrum [8] is remarkable, particularly at high $P^{T}$.

'Soft matrix element corrections' 7,8] refers to correcting emissions generated by the parton shower algorithm using the first order tree level matrix element. Since the HERWIG parton shower ordering variable is an energy-weighted angle which does not necessarily imply ordering in transverse momentum, it is necessary to apply the correction to every emission which has the highest $P^{T}$ so far. The soft corrections are described for the single boson production case in Ref. [8] and were noted to have a small effect in comparison to the hard matrix element corrections for single boson production. The main advantage of the soft corrections is in providing for a smooth transition between the parton shower and Dead Zone regions - though in practice it is difficult to find observables sensitive to this transition. The primary difference with regards to soft corrections for diboson production as compared to the single boson case are the real emission graphs involving a gluon anchored to an internal line, such as the one shown in Figure 11. These graphs are absent for the single boson case and are in no way approximated by the parton shower which traces only external lines. In contrast to the other emission graphs, these graph do not have any collinear singularities. This means that their relative contribution to the cross section is largest in the central regions (since other emission graphs dominate in the collinear regions). Thus, the relative effect of these graphs in the parton shower region is small, and it is more important to account for them in the Dead Zone region where the physics of these graphs may become important.

The present study uses the soft matrix element corrections already implemented in HERWIG for single boson production. Since the emission cross section enters only as a ratio to the Born cross section, the HERWIG implementation accounts for the first order emission from an external line of Feynman graphs for color singlet production to good approximation, but does not account for emissions from an internal line. Implementing the soft corrections for the specific case of $W Z$ production would require intrusive changes to the HERWIG parton shower code. By accepting this approximation, the HERWIG code is kept unmodified and modular. Nevertheless it is recognized that explicit diboson soft matrix element corrections would be a useful extension to this study.

The HERWIG soft matrix element corrections (which are approximate for the diboson case) have very little impact on the distributions presented in this paper. It is expected that the exact soft matrix element corrections (i.e. explicitly including all diboson graphs) would not change the situation in an appreciable way.

\section{A. Matrix element corrected results}

LO and matrix element corrected distributions for hadronic $W Z$ production are obtained by implementing the Born level $n$-body process and tree level $(\mathrm{n}+1$ )-body process (restricted to the Dead Zone) as external processes in HERWIG using the BHO matrix elements. For diboson production, the distribution corresponding to the single vector boson production transverse recoil studied in Ref. [8] is the diboson system recoil $P_{\mathrm{WZ}}^{T}$. A comparison to the NLO result for this distribution is shown in Figure 3 for the LO with parton shower, first order tree level in the Dead Zone, and matrix element corrected results. Away from the low $P^{T}$ region, the shape of the matrix element corrected result agrees well with the NLO prediction from the stand-alone BHO integration package, demonstrating the effectiveness of hard matrix element corrections. There is no contribution from the Dead Zone in the very low $P_{\mathrm{WZ}}^{T}$ region, so the 
Born and matrix element corrected results coincide in this region (Figure 3 , inset). The soft matrix element corrected LO result is indistinguishable from the LO curve and is not shown.

It is more common in the literature to study the $P_{Z}^{T}$ distribution, for example in probing the triple gauge boson vertex for anomalous couplings. This distribution is also sensitive to hard emissions in the Dead Zone, as shown in Figure 1 where, as for the $P_{\mathrm{WV}}^{T}$ spectrum, the shape of the matrix element corrected $P_{\mathrm{Z}}^{T}$ distribution is a good approximation to the NLO result.

Drawbacks in the matrix element corrections approach and fixed order calculation are evident in Figure 3 . The NLO calculation is not reliable in the very low $P^{T}$ region shown in the inset of Figure 3. The NLO prediction shows a dip just below $5 \mathrm{GeV}$ and a very large contribution at zero. These features are not physical and reflect the specific choices of the regularization scheme used in the matrix elements. A different choice of scheme or scheme parameters would yield a very different result in this region.

A disadvantage of the matrix element corrected distributions is the LO normalization in the parton shower region. Since the total matrix element corrected cross section is the sum of the Born level and the integrated Dead Zone cross sections, it depends explicitly on the Dead Zone boundary defined by Eqs. 11 and 2. A change in the boundary would result in a change in the total cross section. The use of NLO calculations everywhere would remove this dependence and provide a more accurate prediction of the total cross section, particularly for multi-TeV colliders where the contribution from the gluon (anti-)quark initial state is substantial. A second drawback of the matrix element corrections is the inability to model NLO shape effects in the parton shower region. The approximate radiation zero, which is partially filled in by NLO corrections, is an example of a distribution which is sensitive to these effects. Observation of this as yet unseen phenomenon will be a goal of future hadron colliders. This distribution is shown in Figure 5, together with the ratio (or $k$-factors) of the matrix element corrected and Born distributions with respect to the NLO distributions. The $k$-factors are largest in the central radiation zero region and the matrix element corrections are successful in greatly reducing this effect. However, a small variation in $k$-factors in the zero rapidity separation region remains after applying the matrix element corrections.

\section{INCORPORATING THE FULL NLO MATRIX ELEMENT}

In the Dead Zone the square of the real emission graphs $\left(\mathcal{M}_{\text {real emission }}^{2}\right)$ is the only contribution to the cross section and the divergent regions of the real emission phase space are excluded. Outside of the Dead Zone the parton shower is better suited at predicting event shapes and exclusive distributions, though it provides no information on the normalization which in showering event generators is normally derived from the LO cross section only. In this region it is preferable to obtain the normalization from the full NLO cross section while preserving the exclusive event structure from the parton shower.

In the parton shower region the square of the Born graphs, the interference of the Born graphs with the one-loop graphs, and the square of the real emissions graphs contribute at NLO,

$$
\mathcal{M}_{\mathrm{NLO}}^{2}=\mathcal{M}_{\text {Born }}^{2}+\mathcal{M}_{\text {Born }} \otimes \mathcal{M}_{\text {one loop }}+\mathcal{M}_{\text {real emission }}^{2}
$$

The first two terms on the right hand side of Eq. 3 are described by n-body kinematics and occupy a point at $\left(\hat{s} / M^{2}, \cos \hat{\theta}_{j}\right)=(1,0)$ of Figure 2. The last term is described by $(\mathrm{n}+1)$-body kinematics and may populate the entire $\hat{s} / M^{2}$ vs. $\cos \hat{\theta}_{j}$ plane. A regularization scheme is necessary to handle the soft, collinear, and ultraviolet divergences which appear when any of the first order contributions are treated alone. The BHO matrix elements use the phase space slicing method [19] (PSS) wherein a small portion (denoted on Figure 2) of the ( $\mathrm{n}+1)$-body phase space (defined by soft and collinear cutoff parameters $\delta_{\mathrm{s}}, \delta_{\mathrm{c}}$ ) is included in the n-body contribution by means of the soft gluon and leading pole approximations.

One way of achieving NLO normalization while gaining sensitivity to the effect of NLO corrections on the $W Z$ system configuration is to assign an event weight for the hard process which is the average NLO cross section (including virtual graphs) across the entire region accessible to the parton shower for a particular choice of the $W Z$ system configuration.

\footnotetext{
2 The BHO matrix elements employ the two parameter phase space slicing method 19 as regularization scheme. The specific choice of cutoff parameters used for Figure 3 are large $\left(\delta_{\mathrm{s}}=0.05, \delta_{\mathrm{c}}=0.01\right.$ see Ref. 10 for a definition of the cutoffs). A smaller choice of parameters would move events out of the zero histogram bin of the Figure 3 inset towards regions of higher $P^{T}$, until eventually the zero bin would become negative. The use of the subtraction method fon for the $P_{\mathrm{Wz}}^{T}$ distribution in the inset of Figure 3 also yields an unphysical negative first histogram bin. This effect serves as a reminder that fixed order perturbation theory is not well suited for predictions of small inclusive $P^{T}$.
} 
Since all of the divergent regions are contained within the parton shower region, this number is well behaved and positive definite - but is difficult to calculate analytically.

This average cross section can be obtained by means of a 2nd stage integration as outlined in Ref. [16]. It is also noted that it is not necessary to obtain this number with a high degree of accuracy - so long as the average of many such integrations (i.e. events) converges to the correct cross section, which is automatic using the Monte Carlo method.

For the present algorithm the jet volume has a simple definition (Eqs. 1 and 2) with the convenient feature that the phase space slicing method jet volume is a subset of this volume. This means the phase space slicing method can be used to achieve a large portion of the jet volume integration and that the result is not sensitive to the specific choice of PSS cutoff parameters which specify the PSS boundary. As such, the maximal PSS cutoffs (which remain within the range of validity of the soft gluon and leading pole approximations) may be used $\left(\delta_{\mathrm{s}}=0.05, \delta_{\mathrm{c}}=0.01\right.$ have been used, see Ref. [10] for a definition of the cutoffs). This is a significant advantage because the subset occupied by the PSS volume is the region where delicate cancellations between the n-body and $(n+1)$-body graphs occur and also where the cross section is peaked.

The 2nd stage integration, which is performed once for each event in the parton shower region, is accomplished as follows. A $W Z$ event with n-body kinematics is generated ( 8 degrees of freedom specify the n-body configuration assuming the vector bosons are taken on shell: e.g. the $W Z$ system mass $M_{\mathrm{WZ}}, W Z$ system rapidity $y_{\mathrm{WZ}}$, the 2 angles defining the vector boson production, and 2 angles for each of the vector boson decays) and defines the $W Z$ system configuration and boost. For a LO showering Monte Carlo event, the Born level cross section would be evaluated and constitute the event weight. Instead, the aim is to evaluate the average cross section of the phase space into which the parton shower may evolve the event.

The NLO n-body cross section is calculated using the BHO matrix element which requires a further 4 degrees of freedom to specify the soft and collinear corrections arising in the PSS method. Thus by integrating over these 4 degrees of freedom the average n-body weight $\left\langle d \sigma^{\mathrm{n}-\text { body }}\right\rangle$ is obtained and accounts for the region inside the PSS boundary (on the left side of the dashed line) in Figure 2. This number may be negative. For the integration of the remainder of the parton shower region an emission is explicitly sampled using 3 additional degrees of freedom (e.g. the $W Z j$ system mass and the 2 angles specifying the emission direction). These 11 degrees of freedom (3 plus 8 from the n-body configuration) specify the $(\mathrm{n}+1)$-body kinematics. It is necessary to use the appropriate overall boost, so as to span the identical phase space the HERWIG parton shower is able to reach from the n-body kinematics. HERWIG applies a conformal boost to the hard process which keeps the hard process mass and rapidity fixed. For the $(\mathrm{n}+1)$-body case the $W Z j$ system rapidity is thus

$$
y_{(\mathrm{n}+1)-\text { body }}=\ln \left(\frac{\sqrt{M_{\mathrm{WV}}^{2}+P_{\mathrm{WZ}}^{T}{ }^{\star 2}}}{E_{\mathrm{WZ}}{ }^{\star}+P_{\mathrm{WZ}}^{z}}\right)+y_{\mathrm{n}-\text { body }}
$$

where $y_{\mathrm{n} \text {-body }}$ is identified as the $W Z$ system rapidity and $P_{\mathrm{WZ}}{ }^{\star}$ is the $W Z$ system 4 -vector in the $W Z j$ frame. The Jacobian for the transformation $y_{\mathrm{n}-\text { body }} \rightarrow y_{(\mathrm{n}+1) \text {-body }}$ is unity. By sampling the 3 degrees of freedom which specify the expansion from n-body to $(\mathrm{n}+1)$-body kinematics and evaluating the NLO matrix element each time (always a positive number), an integration over the remainder of the parton shower region is achieved giving the average $(\mathrm{n}+1)$-body weight $\left\langle d \sigma^{(\mathrm{n}+1)-\text { body }}\right\rangle$.

The cross section for the specific choice of $W Z$ configuration averaged over the parton shower region at NLO is

$$
d \sigma^{\text {parton shower region }}=\left\langle d \sigma^{\text {n-body }}\right\rangle+\left\langle d \sigma^{(\mathrm{n}+1)-\text { body }}\right\rangle
$$

and constitutes the event weight. The input to the parton shower algorithm is the n-body kinematic configuration. The parton shower algorithm (with guidance from the soft matrix element corrections) determines which exclusive emission structure is chosen for the event.

Event generation for the NLO $W Z$ process consists of generating a sample of events in the parton shower region using the 2nd stage integration method discussed above and a second sample of events in the Dead Zone, as already discussed in Sec. II. The event weights for both types of events are positive numbers (unlike for the NLO integration packages which produces events with both positive and negative weights) so the usual event generator strategy of hit-and-miss may be used to obtain unweighted event samples.

\section{A. Results}

The cross sections for the regions of interest are enumerated in Table for $W^{+} Z$ production at LHC with decays to electron/muon-type leptons. The total Born level cross section is almost a ( $k$-)factor 1.5 down from the NLO result. 
The Dead Zone accounts for about $8 \%$ of the total cross section. The sum of the integrated parton shower region cross section and the Dead Zone cross section agrees with the total NLO cross section which serves as a cross check that the parton shower region contribution is correctly evaluated with the 2nd stage integration.

In Figure 6 the transverse recoil of the $W Z$ system is shown for this 2nd stage integration method (which also includes matrix element corrections). The normalization is the same as the NLO normalization by construction. The NLO and matrix element corrected results from Figure 3 are superimposed. In the region of moderate to high $P_{\mathrm{WZ}}^{T}$ the result agrees well with the NLO prediction. In the region of smaller $P_{\mathrm{WZ}}^{T}$, events with low transverse momentum receive extra recoil from multiple emissions and are pushed towards slightly higher $P_{\mathrm{WZ}}^{T}$. At very small $P_{\mathrm{WZ}}^{T}$ (inset) the shape agrees very well with the parton shower distribution and goes smoothly to zero with no dependence on the NLO regularization scheme. The ratio to the fixed NLO distribution is slightly flatter (Fig. 6, bottom) than for the matrix element corrected distribution.

A second distribution which is sensitive to the region of small inclusive jet $P^{T}$ is the azimuthal separation of the vector bosons shown in Figure 7. As the separation approaches $\pi$, the inclusive jet $P^{T}$ goes to zero and the fixed order prediction becomes unreliable, as evidenced by the sharp increase in differential cross section very near $\phi(W)-\phi(Z)=$ $\pi$. The 2nd stage integration and matrix element corrected results do not suffer from this discontinuity and give a smooth, physical prediction across the full range which is higher than the NLO result away from $\phi(W)-\phi(Z)=\pi$. This is an example of a distribution where the NLO prediction is not accurate.

The $Z$ transverse momentum is shown in Figure 8 for the 2nd stage integration method, the matrix element corrections, and the NLO calculation. The 2nd stage integration is in good agreement with the NLO prediction throughout, while the matrix element corrections suffer from normalization problems. The NLO calculation is accurate in the low $P_{\mathrm{Z}}^{T}$ region for this observable since an integration over a large range of inclusive jet $P^{T}$ is automatic for the low $P_{\mathrm{Z}}^{T}$ bins.

The rapidity separation of the vector bosons is shown in Figures 9 for the 2nd stage integration method, the matrix element corrections, and the NLO calculation. The 2nd stage integration is in good agreement with the NLO prediction throughout, with a very slight enhancement visible in the radiation zero region due to multiple emissions. The ratio of the 2nd stage integration distribution to the NLO result is unity and almost constant throughout, whereas the matrix element corrected ratio is about 1.3 with a small variation in the zero separation region. The 2 nd stage integration has accounted for the effects of the first order corrections on the $W Z$ system configuration (including the separation in rapidity) and so this small variation is not present in that distribution.

\section{B. Complications due to negative weights}

For all of the 2nd stage integration method distributions (Figures 65) the integration over the parton shower region (performed once per event) is accomplished with 50 samples of the 3 degrees of freedom which define the expansion to $(\mathrm{n}+1)$-body phase space and 10 samples of the 4 degrees of freedom which define the n-body PSS corrections. The 2nd stage integration increases the computer time required to generate one weighted event with the HERWIG showering event generator by a factor 1.3. This is due mostly to the integration, but also to the added number of mathematical operations necessary to evaluate the NLO matrix element as compared to the LO one.

Since the n-body contribution can be negative, the total event weight (Eq. 5) can be within statistical precision of zero if the integration statistics (number of samples) are small enough and the cancellations (between n-body and $(\mathrm{n}+1)$-body contributions) are large enough. This means that negative events can very occasionally occur (a discussion of 2nd stage integration negative weighted events is given in Ref. 16 ). Negative events of this type are simply discarded. The bias introduced by discarding these negative events is easy to evaluate. The absolute value of the discarded negative weight events is superimposed on Figures 69 as a shaded histogram. These histograms are 5 orders of magnitude below the 2nd stage integration distributions (too small to resolve on Figures 7 and 9) and are largest in the region where the differential cross section is also largest, and so the effect of these negative events is everywhere negligible.

It is possible to use as few as one sample of each type (n-body and (n+1)-body) for the 2nd stage 'integration'. The effect of decreasing the samples in this way is to increase the frequency of negative weight events - in this case the bias increases to $1 \%$, which represents a maximum ceiling for the bias.

\footnotetext{
3 The computation time will increase significantly when generating unweighted events.

${ }^{4}$ The lepton correlation effect discussed in Ref. 16. also occurs in the present study, but the effect is small.
} 
Finally it is noted that an adaptation of the PSS method may eliminate the negative event weights entirely allowing for the unbiased use of a single sample to evaluate the parton shower region cross section. In Ref. [21] a hybrid [22] of the subtraction and the one parameter PSS regularization schemes is investigated. For each n-body configuration the PSS cutoff parameter which gives a zero n-body contribution is calculated - in this manner the virtual and real emission cancellations are enforced and all of the phase space is partitioned to be $(n+1)$-body. One concern with this method is that the minimum jet energy is coupled to the n-body momentum configuration. However, there can be no bias for any observable which is insensitive to the specific PSS boundary for a given n-body configuration. So long as the hybrid PSS region is everywhere contained within the parton shower region (which is expected to be the case), the average cross section of the parton shower region is one such number - and so this method could be employed to evaluate the 2nd stage integration. In this manner the NLO cross section across the parton shower region (Eq. 5) can be represented with a single sample, which with this hybrid method is always a positive number. The weights of many such events will converge to the correct cross section, and event generation would proceed with a single sampling of the phase space, as it normally does for LO showering event generators. The important difference is that in the parton shower region information about the parton emission would be discarded and the n-body kinematic configuration would be used as input for the parton shower, which eliminates the possibility of the jet energy coupling since, as described previously, the parton shower (guided by the soft matrix element corrections) would determine which exclusive event structure is chosen for the event.

\section{CONCLUSIONS}

Matrix element corrections for hadronic diboson production have been implemented following the procedure outlined in Ref. [8] and are interfaced to the HERWIG showering event generator. The corrections are significant in the high- $P^{T}$ regions where the agreement in shape with NLO calculations is significantly improved.

A new method for incorporating the full NLO matrix element is presented. The inclusive recoil against emissions is dominated by the parton shower for events in the soft and collinear regions where the parton shower is expected to give a good description of the physics, and is dominated by the first order matrix element for events in the hard central region where perturbation theory provides a reliable prediction. The normalization is everywhere NLO. Events generated with this method have positive definite event weight, such that unweighted events can be easily produced by means of Monte Carlo hit-and-miss. The method is generalizable to any color singlet production process.

The numerical implementation of the method is straight-forward providing a NLO matrix element (employing the PSS regularization scheme) is available.

The difference in distribution shapes between the matrix element correction approach and the 2nd stage integration incorporating the full NLO matrix element is small - though noticeable for example in distributions such as the diboson rapidity separation. The 2nd stage integration approach has the further advantage of providing NLO normalization.

Diboson event samples from hadron colliders to date are small and insensitive to QCD corrections, so a meaningful comparison to data will have to await Tevatron Run II and LHC.

\section{ACKNOWLEDGMENTS}

The author would like to thank the ATLAS Collaboration and in particular I. Hinchliffe, M. Lefebvre, and J. B. Hansen. I am grateful to U. Baur for providing the matrix elements, encouragement, and informative correspondence. I benefited from discussions with G. Corcella, M. Mangano, G. Ridolfi, M. Seymour, and B. Webber. I thank L. Bourhis, A. Signer, and J. Stirling for organizing and hosting the 2000 IPPP Workshop on Matrix Elements and Parton Showers in Durham. This work has been supported by the Natural Sciences and Engineering Research Council of Canada.

[1] K. O. Mikaelian, Phys. Rev. D 17, 750 (1978); K. O. Mikaelian, M. A. Samuel and D. Sahdev, Phys. Rev. Lett. 43, 746 (1979); R. W. Brown, D. Sahdev and K. O. Mikaelian, Phys. Rev. D 20, 1164 (1979); T. R. Grose and K. O. Mikaelian, Phys. Rev. D 23, 123 (1981); S. J. Brodsky and R. W. Brown, Phys. Rev. Lett. 49, 966 (1982); M. A. Samuel, Phys. Rev. D 27, 2724 (1983); R. W. Brown, K. L. Kowalski and S. J. Brodsky, Phys. Rev. D 28, 624 (1983); R. W. Brown and K. L. Kowalski, Phys. Rev. D 29, 2095 (1984). 
[2] U. Baur, T. Han and J. Ohnemus, Phys. Rev. Lett. 72, 3941 (1994) hep-ph/9403248.

[3] T. Sjostrand, Phys. Lett. B157, 321 (1985).

[4] G. Marchesini and B. R. Webber, Nucl. Phys. B310, 461 (1988).

[5] G. Marchesini, B. R. Webber, G. Abbiendi, I. G. Knowles, M. H. Seymour and L. Stanco, Comput. Phys. Commun. 67, 465 (1992); G. Corcella et al., JHEP0101, 010 (2001) hep-ph/0011363.

[6] T. Sjöstrand, Comput. Phys. Commun. 82, 74 (1994); T. Sjostrand, P. Eden, C. Friberg, L. Lonnblad, G. Miu, S. Mrenna and E. Norrbin, hep-ph/0010017.

[7] M. H. Seymour, Z. Phys. C56, 161 (1992); M. H. Seymour, LU-TP-94-12 Contributed to 27th International Conference on High Energy Physics (ICHEP), Glasgow, Scotland, 20-27 Jul 1994; M. H. Seymour, Comput. Phys. Commun. 90, 95 (1995) hep-ph/9410414; G. Corcella and M. H. Seymour, Phys. Lett. B442, 417 (1998) hep-ph/9809451.

[8] G. Corcella and M. H. Seymour, Nucl. Phys. B565, 227 (2000) hep-ph/9908388].

[9] G. Miu and T. Sjöstrand, Phys. Lett. B449, 313 (1999) hep-ph/9812455; T. Sjostrand, hep-ph/0001032.

[10] J. Ohnemus, Phys. Rev. D44, 3477 (1991); U. Baur, T. Han and J. Ohnemus, Phys. Rev. D51, 3381 (1995) hep$\mathrm{ph} / 9410266$.

[11] S. Frixione, P. Nason and G. Ridolfi, Nucl. Phys. B383, 3 (1992).

[12] J. M. Campbell and R. K. Ellis, Phys. Rev. D60, 113006 (1999) hep-ph/9905386.

13] L. Dixon, Z. Kunszt and A. Signer, Nucl. Phys. B531, 3 (1998) hep-ph/9803250; Phys. Rev. D60, 114037 (1999) [hep$\mathrm{ph} / 9907305$.

[14] Y. L. Dokshitzer, D. Diakonov and S. I. Troian, Phys. Rept. 58, 269 (1980).

[15] T. Han, R. Meng and J. Ohnemus, Nucl. Phys. B384, 59 (1992).

[16] M. Dobbs and M. Lefebvre, Phys. Rev. D 63, 053011 (2001) hep-ph/0011206.

[17] H. L. Lai et al., Phys. Rev. D55, 1280 (1997) hep-ph/9606399].

[18] R.K. Ellis, W.J. Stirling, and B.R. Webber, QCD and Collider Physics (Cambridge University Press, 1996).

[19] H. Baer, J. Ohnemus and J. F. Owens, Phys. Rev. D40, 2844 (1989); B. W. Harris and J. F. Owens, hep-ph/0102128.

[20] R. K. Ellis, D. A. Ross and A. E. Terrano, Nucl. Phys. B178, 421 (1981).

[21] B. Pötter, hep-ph/0007172.

[22] E. W. Glover and M. R. Sutton, Phys. Lett. B342, 375 (1995) hep-ph/9410234.

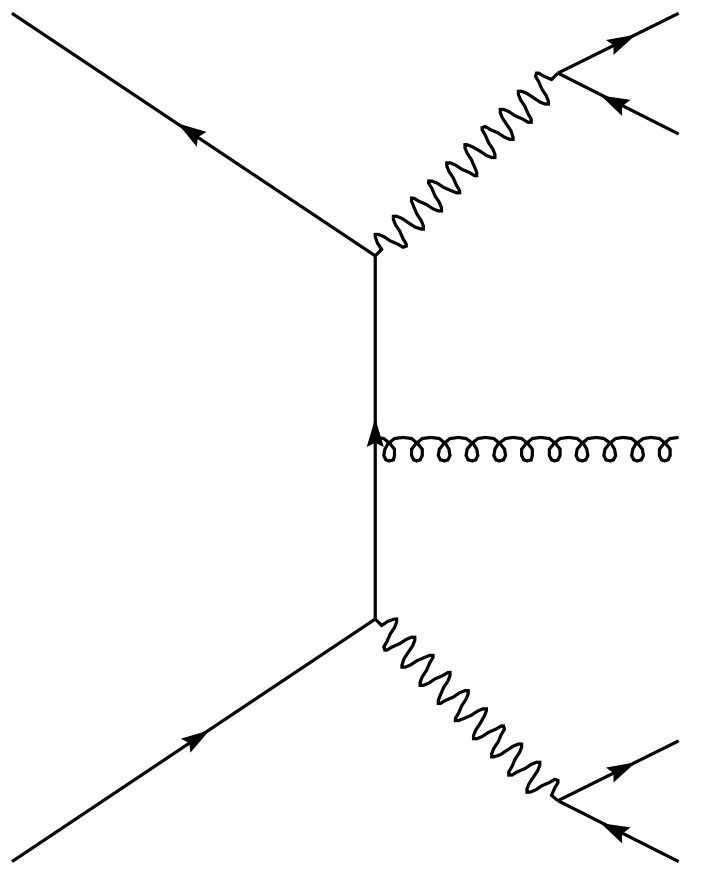

FIG. 1. Example of a first order $W Z$ production graph with a gluon anchored to an internal line. 


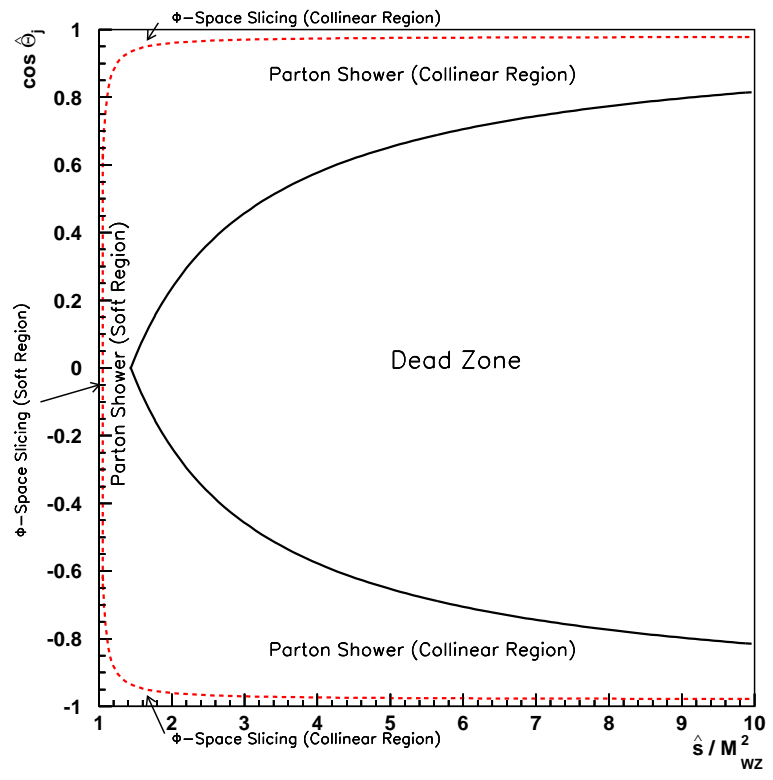

FIG. 2. The $\hat{s} / M^{2}$ vs. $\cos \hat{\theta}_{j}$ plane is shown with the boundary between the complimentary parton shower and Dead Zone regions denoted with a solid line. The phase space slicing region is a subset of the parton shower region and is denoted with a dashed line (for the specific phase space slicing parameters $\delta_{\mathrm{s}}=0.05, \delta_{\mathrm{c}}=0.01$ ).
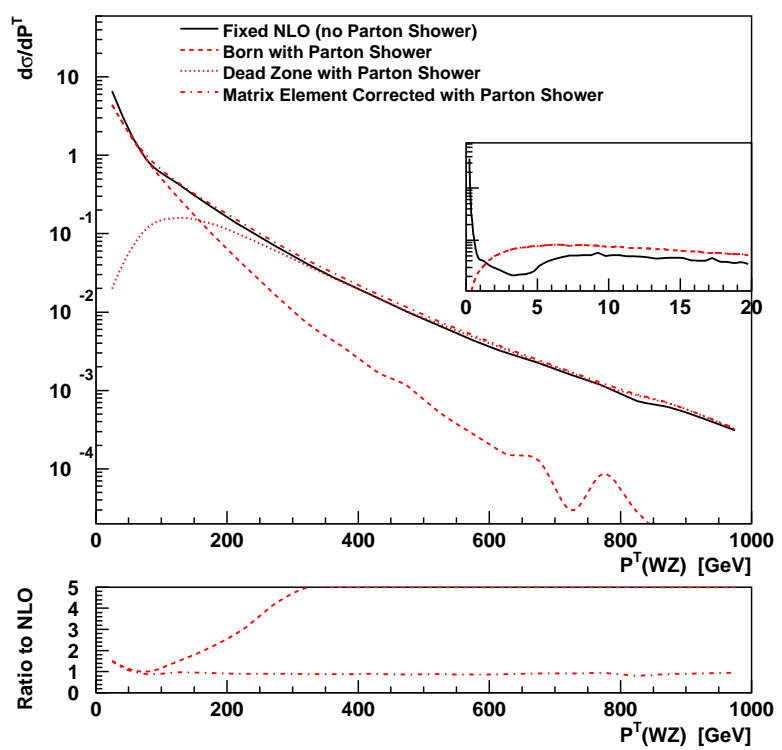

FIG. 3. The transverse momentum distribution of the $W^{+} Z$ system (inclusive recoil against emissions) $P_{\mathrm{WZ}}^{T}$ is compared at NLO, LO with parton shower, first order tree level in the Dead Zone, and at LO with soft and hard matrix element corrections. An enhanced view of the first low $P_{\mathrm{WZ}}^{T}$ bin is shown in the inset and the ratio of the NLO distribution to the LO and matrix element corrected distributions is shown at bottom. 

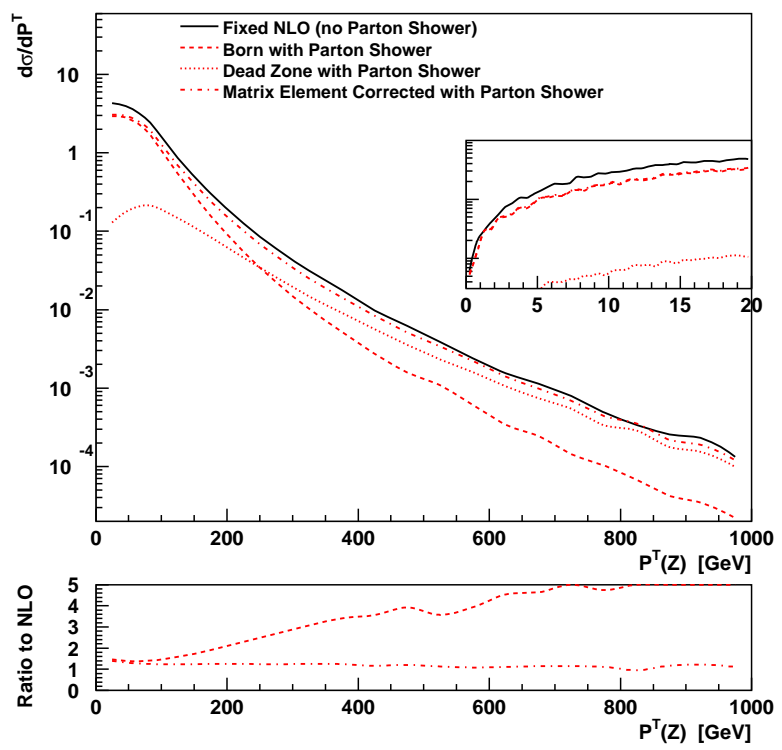

FIG. 4. The transverse momentum distribution of the $Z$-boson $P_{\mathrm{Z}}^{T}$ is compared at NLO, LO with parton shower, first order tree level in the Dead Zone, and at LO with soft and hard matrix element corrections. An enhanced view of the first low $P_{Z}^{T}$ bin is shown in the inset and the ratio of the NLO distribution to the LO and matrix element corrected distributions is shown at bottom.

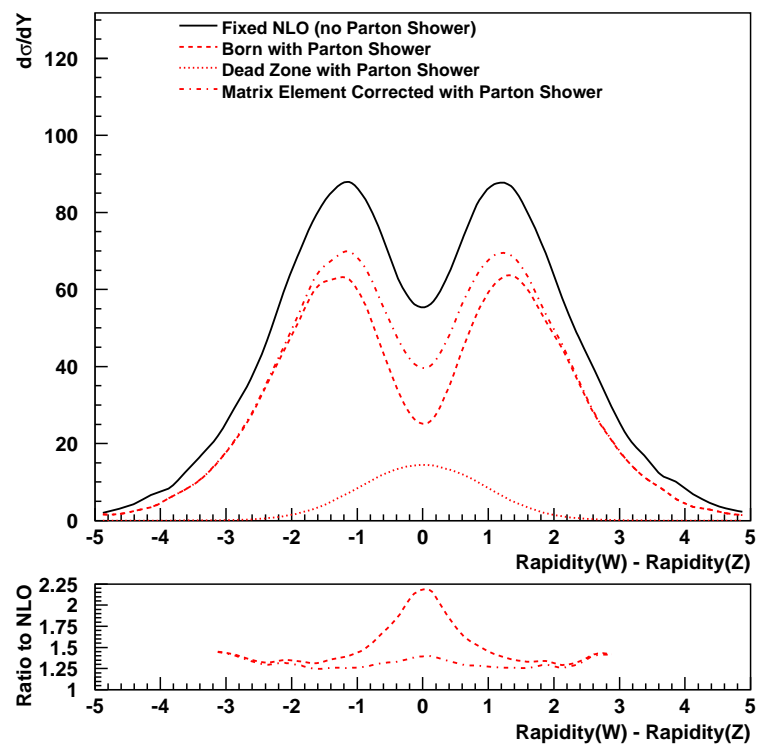

FIG. 5. The rapidity separation distribution of the $W^{+}$and $Z$-bosons is compared at NLO, LO with parton shower, first order tree level in the Dead Zone, and at LO with soft and hard matrix element corrections. The dip in the zero separation region is the approximate radiation zero. The ratio of the NLO distribution to the LO and matrix element corrected distributions is shown at bottom. 


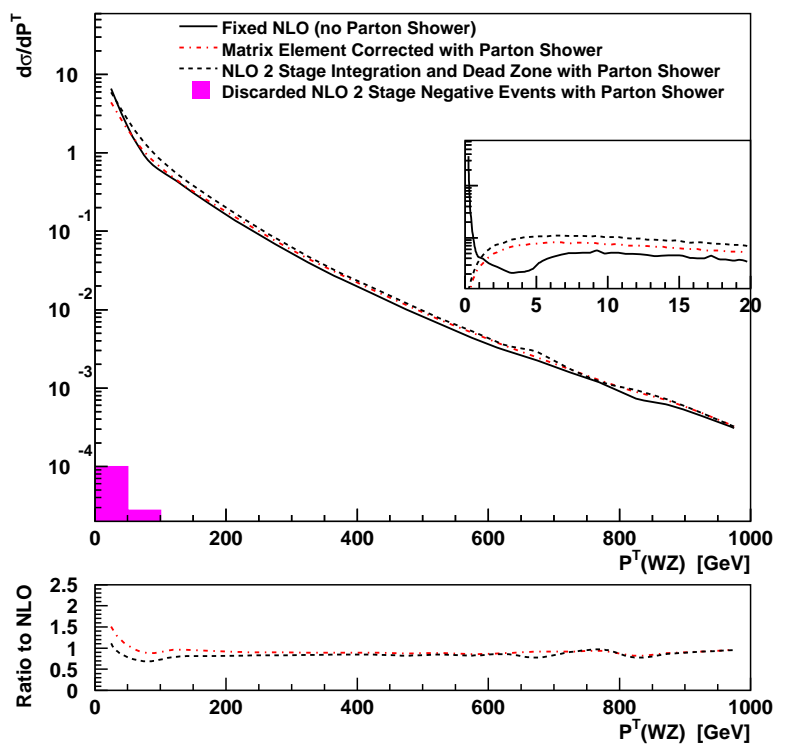

FIG. 6. The transverse momentum distribution of the $W^{+} Z$ system (inclusive recoil against emissions) $P_{\mathrm{WZ}}^{T}$ is compared at $\mathrm{NLO}$, at LO with soft and hard matrix element corrections, and using the 2nd stage integration (which also employs soft and hard matrix element corrections). An enhanced view of the first low $P_{\mathrm{WZ}}^{T}$ bin is shown in the inset and the ratio of the NLO distribution to the matrix element corrected and 2nd stage integration distributions is shown at bottom.

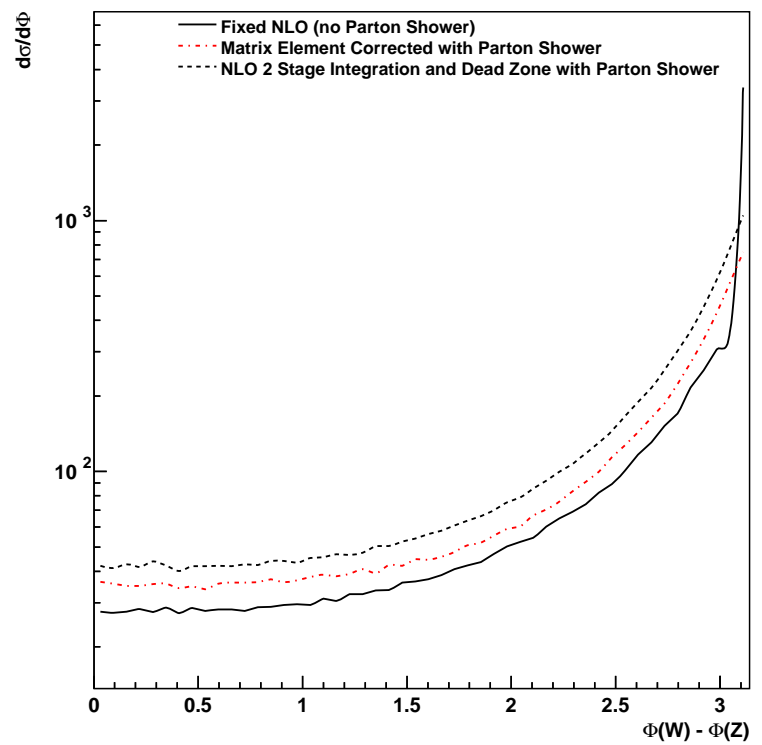

FIG. 7. The azimuthal vector boson separation $\phi\left(W^{+}\right)-\phi(Z)$ distribution is compared at NLO, at LO with soft and hard matrix element corrections, and using the 2nd stage integration (which also employs soft and hard matrix element corrections). 


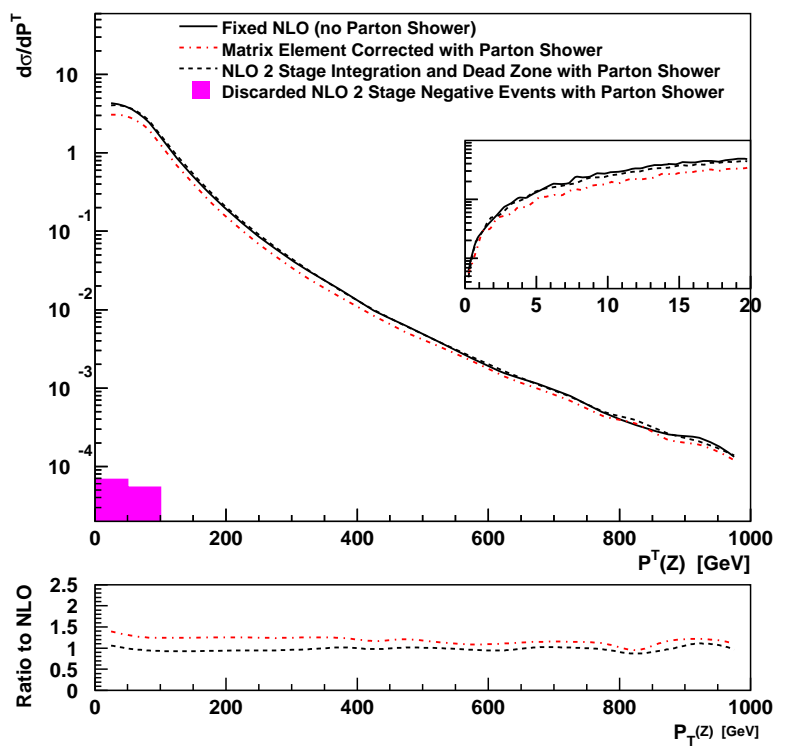

FIG. 8. The transverse momentum distribution of the $Z$-boson $P_{\mathrm{Z}}^{T}$ is compared at NLO, at LO with soft and hard matrix element corrections, and using the 2nd stage integration (which also employs soft and hard matrix element corrections). An enhanced view of the first low $P_{\mathrm{Z}}^{T}$ bin is shown in the inset and the ratio of the NLO distribution to the matrix element corrected and 2nd stage integration distributions is shown at bottom.

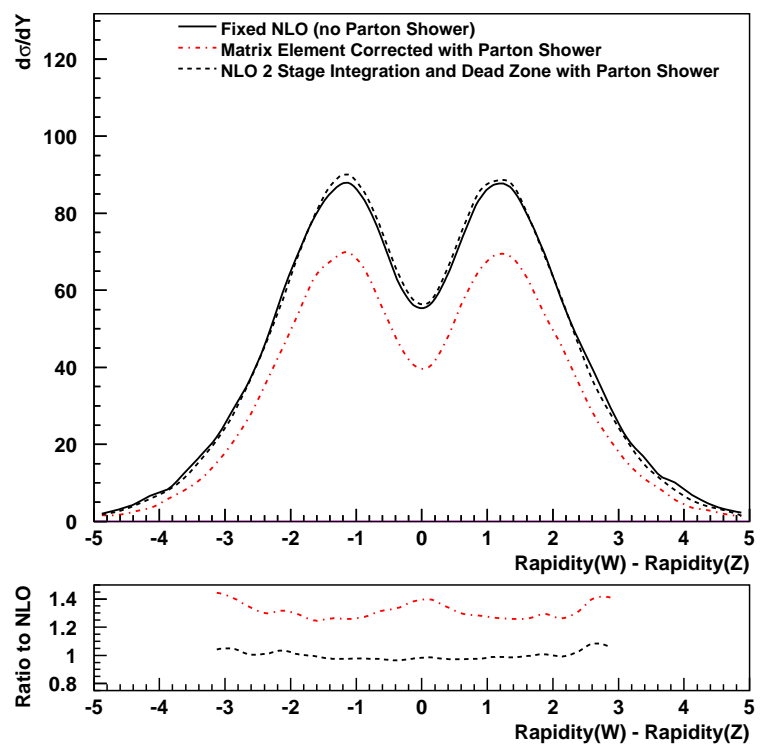

FIG. 9. The rapidity separation distribution of the $W^{+}$and $Z$-bosons is compared at NLO, at LO with soft and hard matrix element corrections, and using the 2nd stage integration (which also employs soft and hard matrix element corrections). The dip in the zero separation region is the approximate radiation zero. The ratio of the NLO distribution to the matrix element corrected and 2nd stage integration distributions is shown at bottom. 
TABLE I. The integrated cross section for $W^{+} Z$ production with decays to electron/muon-type leptons at LHC evaluated using the BHO matrix elements is tabulated. The ratio (or $k$-factor) of the NLO calculation to the Born is about 1.48 , which improves to 1.32 when the Dead Zone region is added to the Born result. The ratio for the NLO calculation to the sum of the 2nd stage integration and the Dead Zone is unity by construction.

\begin{tabular}{lc}
\hline \hline Born & $288.2 \pm 0.3 \mathrm{fb}$ \\
Dead Zone region (NLO) & $34.43 \pm 0.02 \mathrm{fb}$ \\
2nd stage integration over parton shower region (NLO) & $391.8 \pm 0.3 \mathrm{fb}$ \\
NLO (all phase space included) & $426.0 \pm 0.5 \mathrm{fb}$ \\
\hline \hline
\end{tabular}

